\title{
The power of new experimental techniques in astronomy: zooming in on the black hole in the Center of the Milky Way
}

\author{
Reinhard Genzel \\ Max-Planck Institut für extraterrestrische Physik (MPE), Garching, FRG \\ and \\ Department of Physics, University of California, Berkeley, USA \\ email: genzel@mpe.mpg.de
}

\begin{abstract}
It is becoming ever easier to obtain first class astronomical data by working solely from one's computer terminal, the modern equivalent of the armchair. This wonderful development may tempt us to forget that astronomical discoveries and findings are first and foremost driven by hard-won progress in observational and experimental capabilities. The present article is meant to demonstrate this assertion by reviewing recent developments and future possibilities in studying the Center of the Milky Way, our best case for the existence of a (massive) black hole and a superb laboratory for studying the physical processes in the immediate vicinity of such an enigmatic object.
\end{abstract}

Keywords. Galaxy: center, infrared: stars, stars: individual (Sgr A*)

\section{Black Holes}

Rev. John Michell was in 1784 the first to note that a sufficiently compact star may have a surface escape velocity exceeding the speed of light. He argued that an object of the mass of the Sun (or larger) but with a radius of $3 \mathrm{~km}$ would thus be invisible. A proper mathematical treatment of this problem then had to await Albert Einstein's General Relativity (Einstein 1916). Karl Schwarzschild's solution of the vacuum field equations in spherical symmetry (Schwarzschild (1916)) demonstrated the existence of a characteristic event horizon, the Schwarzschild radius $R_{s}=2 G M / c^{2}$, within which no communication is possible with external observers. Kerr (1963) generalized this solution to spinning black holes. The mathematical concept of a black hole was established (although the term itself was coined only later by John Wheeler (1968). But are these theoretical objects of General Relativity realized in Nature?

Astronomical evidence for the existence of black holes started to emerge in the 1960s with the discovery of distant luminous quasars (QSOs: Schmidt 1963) and variable X-ray emitting binaries in the Milky Way (Giacconi et al. 1962). Early on it became clear from energetic arguments that the enormous luminosities and energy densities of QSOs can most plausibly be explained by accretion of matter onto massive black holes (Lynden-Bell 1969; Rees 1984). We now know from high resolution imaging that QSOs are located (without exception) at the nuclei of large, massive galaxies. QSOs just represent the most extreme and spectacular among the general nuclear activity of most galaxies. This includes variable X-ray and $\gamma$-ray emission and highly collimated, relativistic radio jets, all of which cannot be plausibly accounted for by stellar activity. It is thus tempting to conclude that most galactic nuclei may harbor massive black holes. 


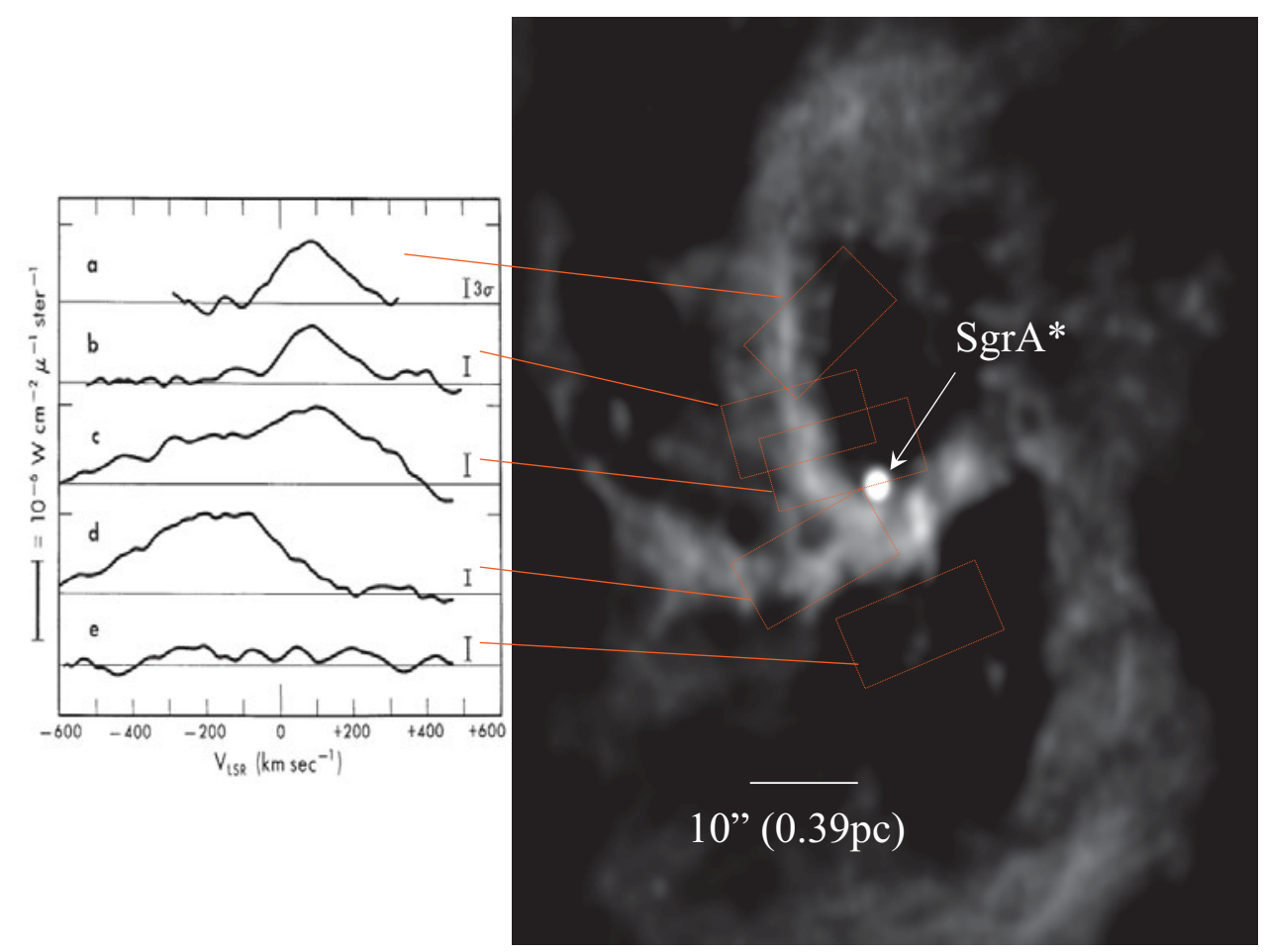

Figure 1. Right: $3.6 \mathrm{~cm}$ VLA radio continuum map of the central parsec (Roberts \& Goss 1993). Left: $12.8 \mu \mathrm{m}$ [Ne II] line profiles (Wollman et al. 1977) for the apertures indicated on the radio map. The radio emission delineates ionized gas streamers (the 'mini-spiral') orbiting the compact radio source Sgr A*. The Wollman et al. observations provided the first dynamic evidence from large gas velocities that there might be a hidden mass of 3-4 million $\mathrm{M}_{\odot}$ located near Sgr A*.

In the case of Galactic X-ray stellar binary systems (see Giacconi, 2003, for a historic account) dynamical mass determinations from Doppler spectroscopy of the visible primary star have established in a number of cases that the mass of the X-ray emitting secondary is significantly larger than the maximum stable neutron star mass of about $3 \mathrm{M}_{\odot}$ (McClintock \& Remillard 2006). These X-ray sources thus are excellent candidates for stellar black holes. To apply similar, direct dynamical mass determinations to massive black hole candidates it is necessary to determine the motions of test particles (interstellar gas or stars) in close orbit around the nucleus. The aim is to show from measurements at different separations from the center that the gravitational potential is dominated by a compact, non-stellar mass that cannot be in any other form than that of a massive black hole. Such measurements are not possible (yet) in distant QSOs but have become feasible in nearby galaxy nuclei, where solid evidence for central dark mass concentrations in about 30 galaxies has emerged over the past two decades (Kormendy 2004), from Hubble Space Telescope imaging and spectroscopy and from very long baseline radio interferometry (VLBI).

The first really convincing case that these dark mass concentrations cannot just be dense conglomerates of white dwarfs, neutron stars and perhaps stellar black holes, emerged in the mid 1990s from spectacular VLBI observations of the nucleus of NGC 4258, 

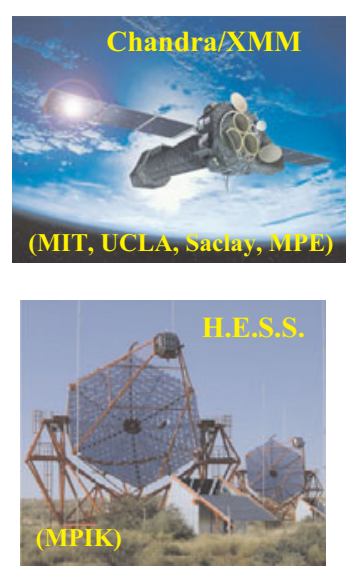
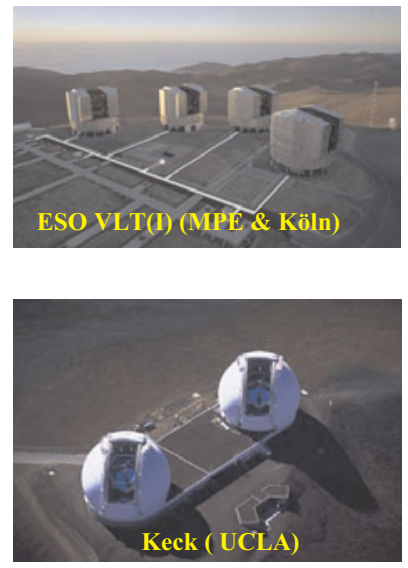
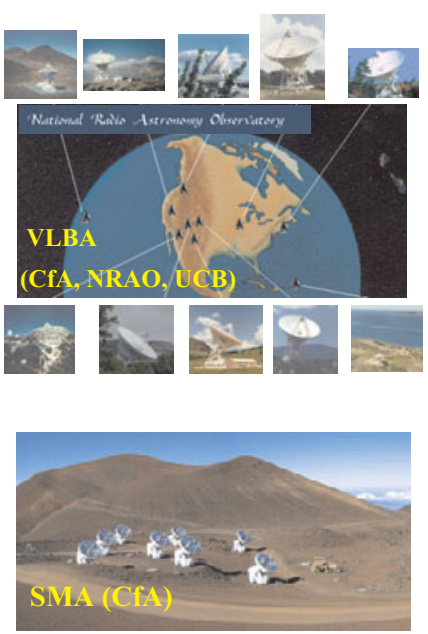

Figure 2. Some the key facilities that have contributed to Galactic Center research over the past two decades. Lower left: the TeV-Gamma Ray telescope HESS. (High Energy Stereoscopic System) in Namibia. Top left: the X-ray telescopes XMM-Newton (ESA) and Chandra (NASA). Center: large optical/near-infrared telescopes, such as the Keck telescopes and the ESO VLT. Top right: the Very Long Baseline Array (NRAO). Bottom right: The Submillimeter Array. The main institutions/groups involved in Galactic Center research with these facilities are listed.

a mildly active galaxy at a distance of $7 \mathrm{Mpc}$ (Miyoshi et al. 1995). The VLBI observations showed that the galaxy nucleus contains a thin, slightly warped disk of $\mathrm{H}_{2} \mathrm{O}$ masers in Keplerian rotation around a point mass of 40 million $\mathrm{M}_{\odot}$. The inferred effective density of this mass exceeds a few $10^{9} \mathrm{M}_{\odot} \mathrm{pc}^{-3}$ and thus cannot be a long-lived cluster of 'dark' astrophysical objects of the type mentioned above.

Over the past decade, measurements of stellar orbits in the nearest galaxy nucleus, namely the Center of the Milky Way, have now shown that the central dark mass concentration in the Galactic Center must indeed be a massive black hole, beyond any reasonable doubt.

\section{Sagittarius $A^{*}$}

The central light years of our Galaxy contain a dense and luminous star cluster, as well as several components of neutral, ionized and extremely hot gas (Genzel et al. 1994). In the late 1970s and early 1980s, Professor Charles Hard Townes, Nobel Laureate for the invention of the maser and laser, with his outstanding group of students and postdocs at the University of California, made the discovery, with the then emerging technique of mid-infrared spectroscopy, that the nucleus of our Milky Way contains a non-stellar, central mass concentration of 3-4 million $\mathrm{M}_{\odot}$ (Fig. 1). The Berkeley group concluded that this mass concentration might be a massive black hole (Wollman et al. 1977; Townes et al. 1982). The Galactic Center also contains a very compact radio source, Sgr A* (Figs. 1 and 3; Balick \& Brown 1974), which is located at the center of the nuclear star cluster and ionized gas. Short-wavelength centimeter and millimeter VLBI observations 

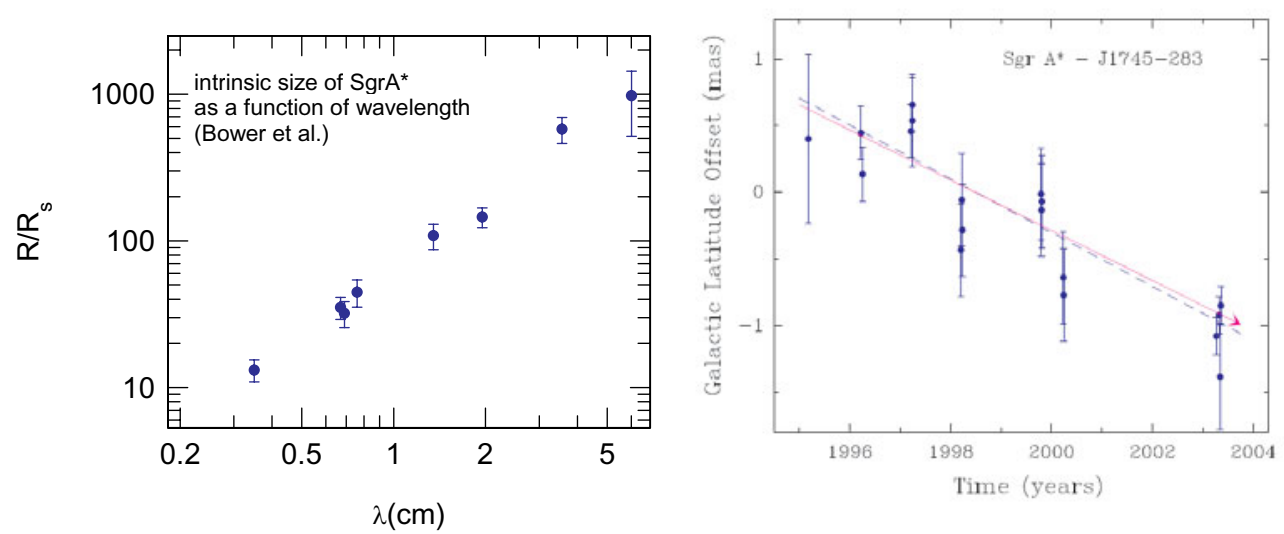

Figure 3. Radio characteristics of Sgr A*. Left: Inferred intrinsic major axis size of the radio source as a function of wavelength. The intrinsic sizes are in units of the Schwarzschild radius of a 3-4 million $\mathrm{M}_{\odot}$ black hole (Bower et al. 2004; Bower, private communication). Right: Galactic latitude position residuals of Sgr A* relative to the QSO J1745-283. The dashed line is the variance-weighted best-fit proper motion of $-0.202 \pm 0.019$ milli-arcseconds/yr, or $-7.6 \pm 0.7 \mathrm{~km} \mathrm{~s}^{-1}$ for $R_{\circ}=8.0 \mathrm{kpc}$. The solid arrow indicates the apparent motion of Sgr A* expected for the $7.17 \mathrm{~km} \mathrm{~s}^{-1}$ motion of the Sun perpendicular to the plane of the Galaxy. The difference translates into a $2 \sigma$ upper limit of the residual motion of Sgr A* in the Galactic Center reference frame of less than $18.8 \mathrm{~km} \mathrm{~s}^{-1}$ (from Reid \& Brunthaler 2004).

have established that its intrinsic radio size is a mere 10 light minutes (Fig. 3; Bower et al. 2004; Shen et al. 2005). Sgr A* is also an X-ray emission source, albeit of only modest luminosity (e.g., Baganoff et al. 2001). Most recently, Aharonian et al. (2004) have discovered a source of $\mathrm{TeV} \gamma$-ray emission within $10^{\prime \prime}$ of $\mathrm{Sgr} \mathrm{A}^{*}$.

It is not yet clear whether these most energetic $\gamma$-rays come from Sgr A* itself, or whether they are associated with the nearby supernova remnant Sgr A East. Sgr A* thus may be a supermassive black hole analogous to QSOs, albeit of much lower mass and luminosity. Because of its proximity - the distance to the Galactic Center is about $10^{5}$ times closer than the nearest quasars - high-resolution observations of the Milky Way nucleus offer the unique opportunity of stringently testing the black hole paradigm and of studying stars and gas in the immediate vicinity of a black hole, at a level of detail that will not be accessible in any other galactic nucleus in the foreseeable future. Since the Center of the Milky Way is highly obscured by interstellar dust particles in the plane of the Galactic disk, observations in the visible are not possible. Investigations require measurements at longer wavelengths, in the infrared and microwave bands, or at shorter wavelengths, at hard X-ray and $\gamma$-rays, where the veil of dust is transparent. The dramatic progress in our knowledge of the Galactic Center over the past two decades is a direct consequence of the development of novel facilities, instruments and techniques across the whole range of the electromagnetic spectrum (Fig. 2). 


\section{speckle (1993)}

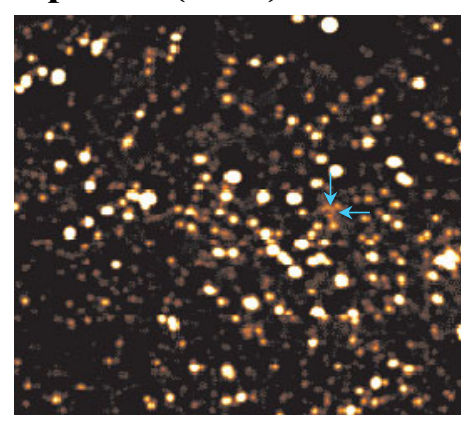

seeing limited (1990)

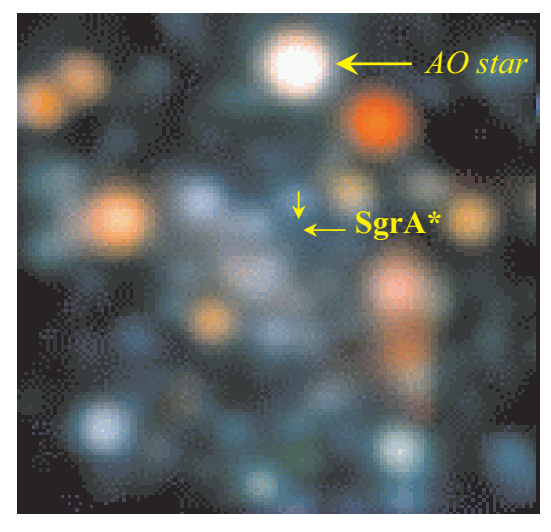

$10 "(0.39 \mathrm{pc})$
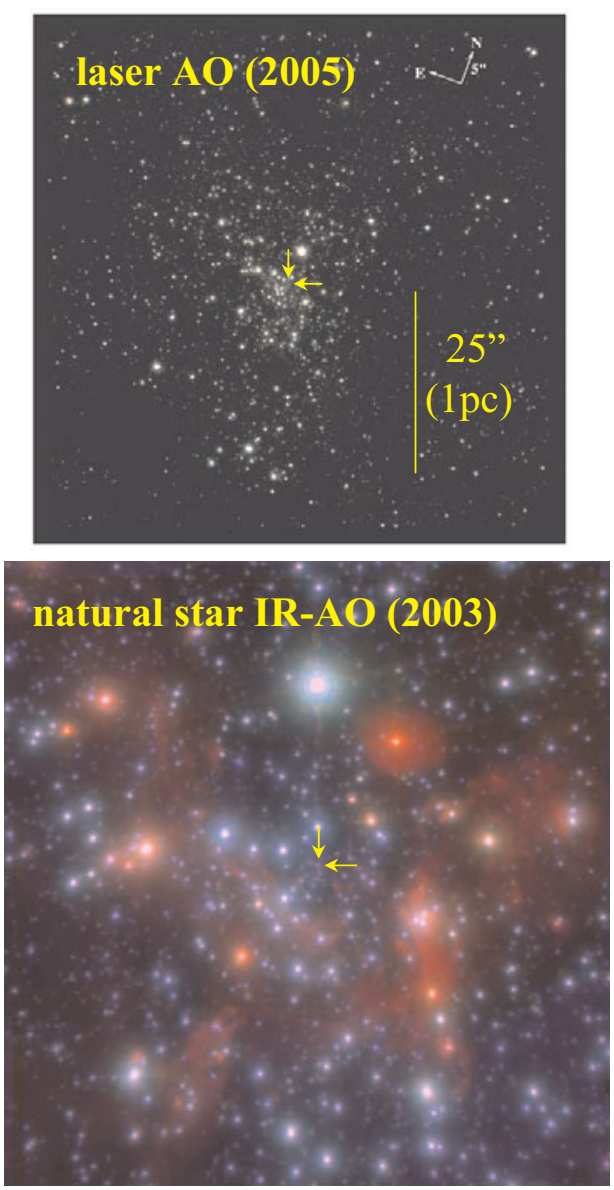

Figure 4. Progress in high-resolution near-infrared imaging of the central few light years of our Milky Way. Lower left: a 'atmospheric seeing' limited $\left(\sim 0.5^{\prime \prime}\right.$ resolution $)$ image in three colors (1.6/2.2/3.5 $\mu \mathrm{m}$ : DePoy \& Sharp (1991)). Top left: a 'cleaned' shift-and-add speckle $2.2 \mu \mathrm{m}$ image at $0.15^{\prime \prime}$ resolution (ESO NTT) from the work of (Eckart et al. 1993, 1995). Bottom right: the same region (at $1.6 / 2.2 / 3.7 \mu \mathrm{m}$ ) at the diffraction limit of the $8 \mathrm{~m}$ ESO VLT $(\sim 0.05 \mu)$, taken with the NACO AO-camera and an infrared wavefront sensor (Genzel et al. 2003b). All these three images are on the same spatial scale. Top right: a Keck diffraction limited image of a $\sim 3$ times larger field, this time with a sodium laser guide star for AO correction (Ghez et al. 2005b). The diffraction limited images are much sharper and also much deeper than the seeing limited images. In addition to increasing the sky coverage the laser allows easy mosaicing of a large field of view. The arrows denote the position of the compact radio source Sgr A* and the location of the AO-star IRS 7. NACO is a collaboration between ONERA (Paris), Observatoire Paris, Observatoire Grenoble, MPE (Garching) and MPIA (Heidelberg) (Lenzen et al. 1998; Rousset et al. 1998). The Keck AO camera NIRC2 was developed at Caltech (Matthews et al. 2007) and the Keck laser system was developed by a consortium of Lawrence Livermore National Laboratory and the Keck Observatory (Wizinovich et al. 2007).

\section{High angular resolution astronomy}

The key obviously lies in very high angular resolution measurements. The Schwarzschild radius of a 3.6 million solar mass black hole at the Galactic Center subtends a mere $10^{-5}$ arcseconds. For high-resolution imaging from the ground it is necessary to correct for the 


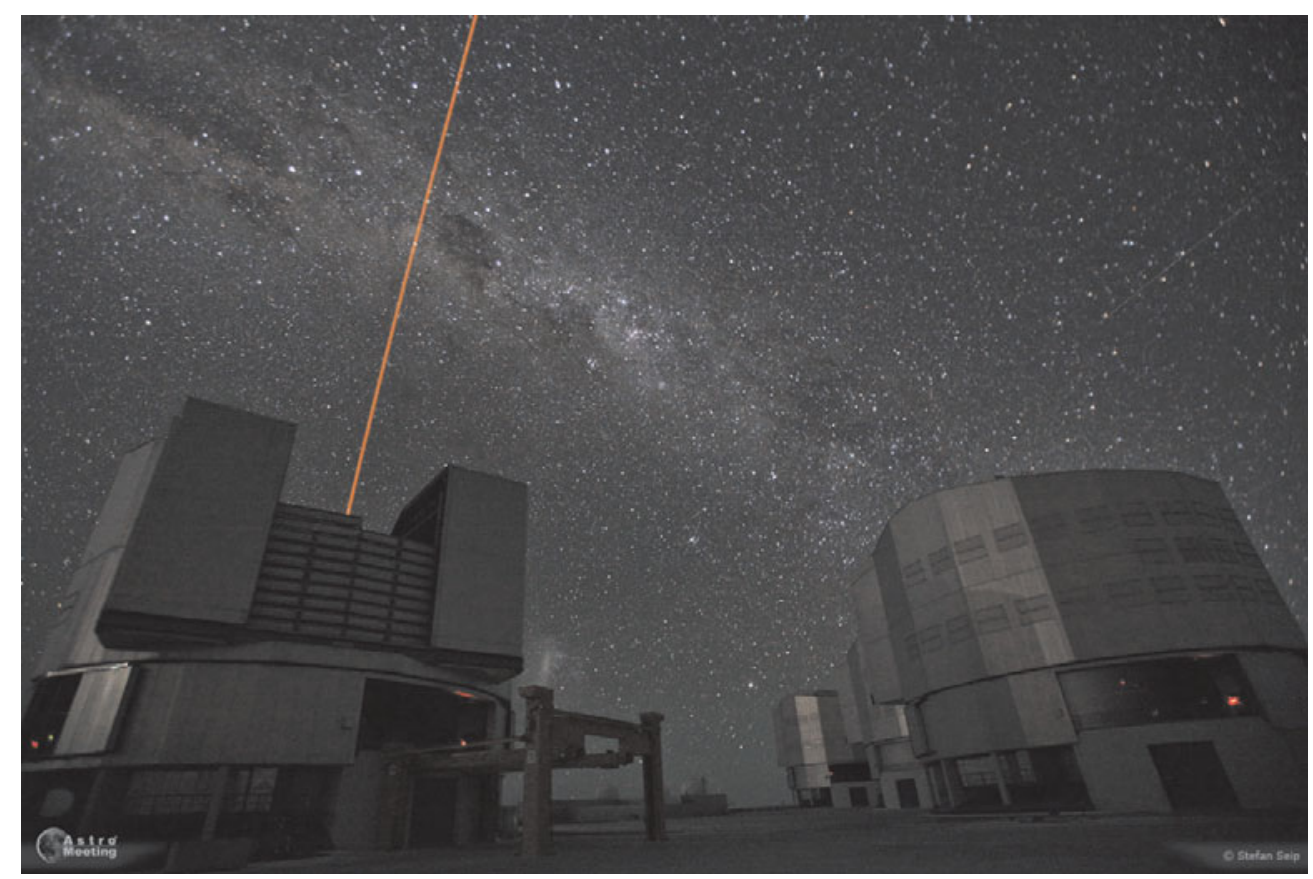

Figure 5. Image of a Na $589 \mathrm{~nm}$ laser beacon (PARSEC) projected from UT4 of the ESO VLT in Chile. The laser beam is focused on a layer of atomic sodium in the upper atmosphere at $\sim 90 \mathrm{~km}$ altitude where it creates an artifical laser 'star' by resonant backscattering. The wavefront of the laser star traveling back from the sky to the telescope can then be used for adaptive optics correction. In the background one can see the band of the southern Milky Way, as well as the Large Magellanic Cloud (close to the ground near UT4). The VLT laser guide star facility is a collaboration between ESO (Garching), MPE (Garching) and MPIA (Heidelberg).

distortions of an incoming electromagnetic wave by the refractive and dynamic Earth atmosphere. VLBI overcomes this hurdle by phase-referencing to nearby QSOs; submilli-arcsecond resolution can now be routinely achieved. In the optical/near-infrared waveband the atmosphere smears out long-exposure images to a diameter at least ten times greater than the diffraction limited resolution of large ground-based telescopes (Fig. 4). From the early 1990s onward initially 'speckle imaging' (recording short exposure images, which are subsequently processed and co-added to retrieve the diffraction limited resolution) and then later 'adaptive optics' (AO: correcting the wave distortions on-line) became available. With these techniques it is possible to achieve diffraction limited resolution on large ground-based telescopes. In the case of AO (Beckers 1993) the incoming wavefront of a bright star near the source of interest is analyzed, the necessary corrections for undoing the aberrations of the atmosphere are computed (on time scales shorter than the atmospheric coherence time of a few milli-seconds) and these corrections are then applied to a deformable optical element (e.g., a mirror) in the light path. The requirements on the brightness of the $\mathrm{AO}$ star and on the maximum allowable separation between star and source are quite stringent, resulting in a very small sky coverage of 'natural star' AO. Fortunately, in the Galactic Center there is a bright infrared star only $6^{\prime \prime}$ away from Sgr A*, such that good AO correction can be achieved with an infrared wavefront sensor system (see lower left inset of Fig. 4). Artificial laser beacons can 


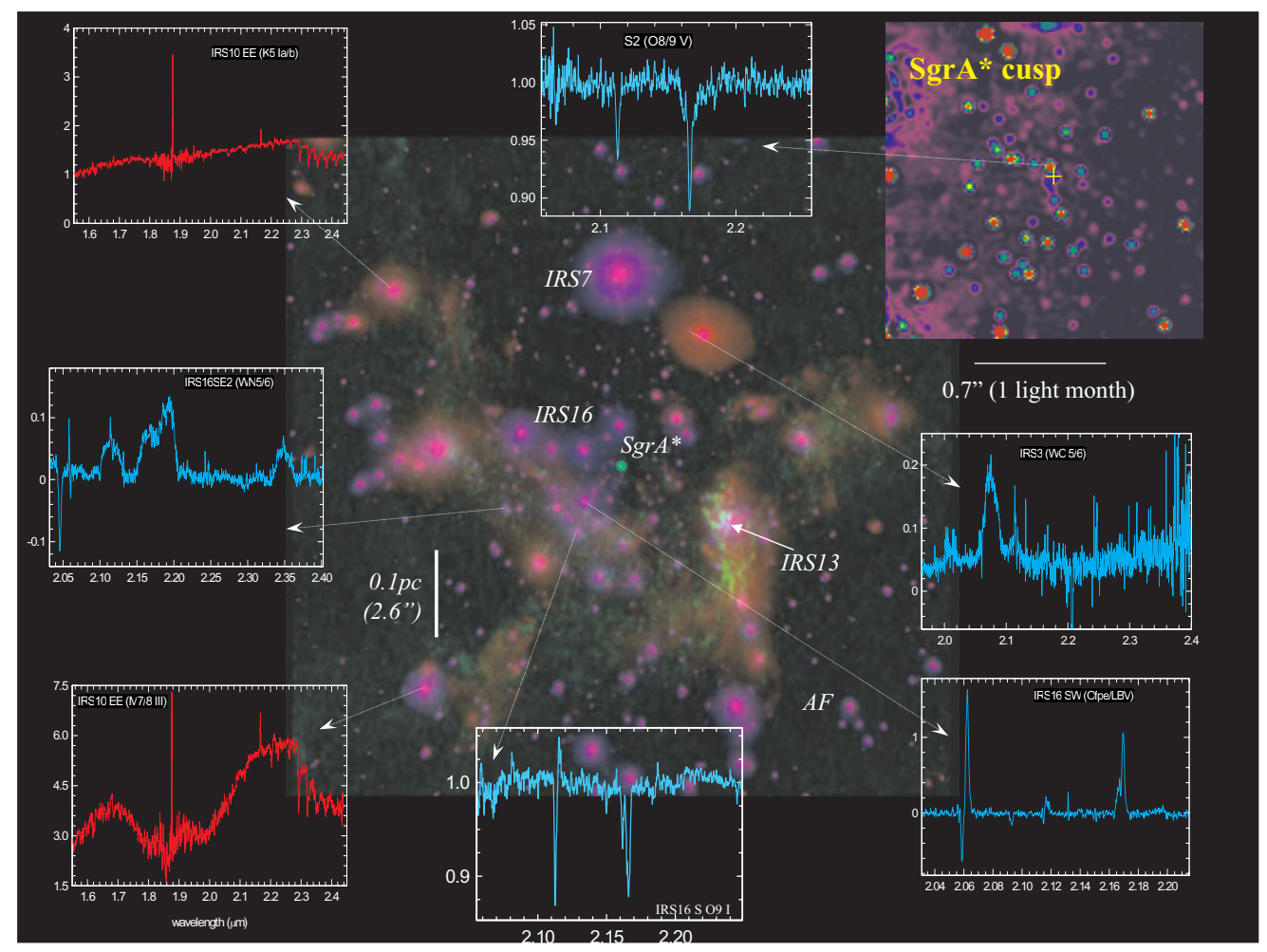

Figure 6. Center: composite of $2.2 \mu \mathrm{m}$ ( $=K$ : blue), $3.7 \mu \mathrm{m}\left(L^{\prime}:\right.$ red) and $1.3 \mathrm{~cm}$ (green) continuum emission ( NACO/VLT \& VLA), probing stars of different types, hot dust, ionized gas and the compact radio source Sgr A*. Top right: 40 milli-arcsecond $1.6 \mu \mathrm{m}$ image (VLT) of the dense central stellar cusp around Sgr A* (cross). Surrounding: selected $2 \mu \mathrm{m}$ spectra of late-type (red) and early-type (light blue) stars in the nuclear cluster taken with the AO assisted integral field spectrometer SINFONI on the VLT (Eisenhauer et al. 2003b; Bonnet et al. 2004).

overcome the sky coverage problem to a considerable extent. For this purpose, a laser beam is projected from the telescope into the upper atmosphere and the backscattered laser light can then be used for AO correction (Fig. 5). The Keck telescope team has already begun successfully exploiting the new laser guide star technique for Galactic Center research (Ghez et al. 2005b).

After AO correction, the images are an order of magnitude sharper and also much deeper than in conventional seeing limited measurements (Figs. 4, 6). The combination of AO techniques with advanced imaging and spectroscopic instruments (e.g., 'integral field' imaging spectroscopy) have resulted in a major breakthrough in high-resolution studies of the Galactic Center.

\section{Nuclear star cluster and 'paradox of youth'}

Looking at the radio/IR composite image in Fig. 6 one of the immediate surprises is the fairly large number of bright stars, a number of which were already apparent on the discovery infrared images of Becklin \& Neugebauer (1975): IRS 7, 13, 16. High-resolution 

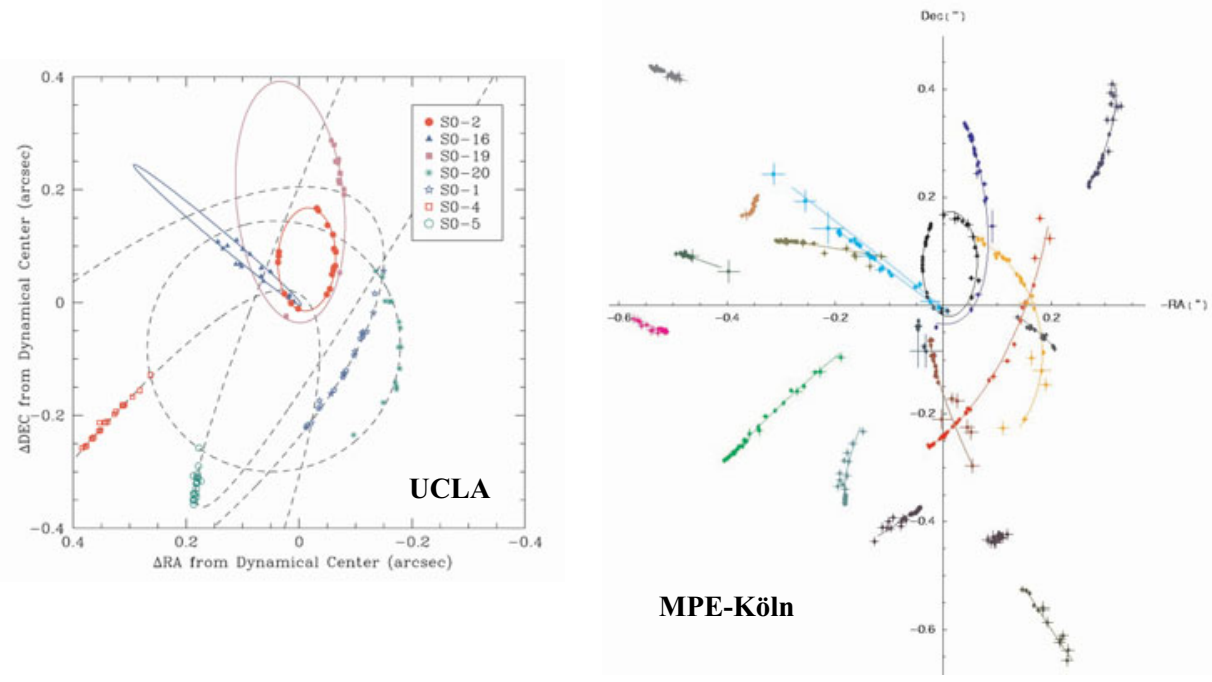

Figure 7. Positions on the sky as a function of time of the central stars ('S'-stars) orbiting the compact radio source Sgr A*. Left inset: the data from the UCLA group working with the Keck telescope (Ghez et al. 2005a). Right inset: the data from the MPE-Cologne group at the ESO-VLT (Schödel et al. 2003; Gillessen et al., in preparation).

infrared spectroscopy reveals that many of these bright stars are somewhat older, latetype supergiants and AGB stars. Starting with the discovery of the 'AF'-star (Fig. 6 lower right; Allen et al. 1990; Forrest et al. 1987), however, an ever increasing number of these bright stars has been identified as being young, massive and early-type. The most recent count from deep SINFONI integral field spectroscopy yields about 100 OB-type stars, including various luminous blue supergiants and Wolf-Rayet stars, but also normal main-sequence OB-type stars (Paumard et al. 2006). The nuclear star cluster is one of the richest concentrations of young massive stars in the Milky Way.

The deep adaptive optics images also trace the surface density distribution of the fainter stars, to about $K \simeq 17-18 \mathrm{mag}$, corresponding to late $\mathrm{B}$ or early A stars (mass 3 $\left.6 \mathrm{M}_{\odot}\right)$, which are a better probe of the density distribution of the overall mass density of the star cluster. While the surface brightness distribution of the star cluster (dominated by the bright stars) is not centered on $\mathrm{Sgr} \mathrm{A}^{*}$, the surface density distribution is. There is clearly a cusp of stars centered on the compact radio source (Fig. 6, upper right inset; Genzel et al. 2003b; Schödel et al. 2007). The inferred volume density of the (observable) cusp is a power-law scaling with $R^{-1.4 \pm 0.1}$. This is quite consistent with the expectation for a stellar cusp around a massive black hole (Alexander 2005).

If there is indeed a central black hole associated with $\mathrm{Sgr} \mathrm{A}^{*}$ the presence of so many young stars in its immediate vicinity constitutes a significant puzzle (Allen \& Sanders 1986; Morris 1993; Alexander 2005). For gravitational collapse to occur in the presence of the tidal shear from the central mass, gas clouds have to be denser than $\sim 10^{9}\left(R /\left(10^{\prime \prime}\right)\right)^{-3}$ hydrogen atoms per $\mathrm{cm}^{3}$. This 'Roche' limit exceeds the density of any gas currently observed in the central region. Recent near-diffraction limited AO 


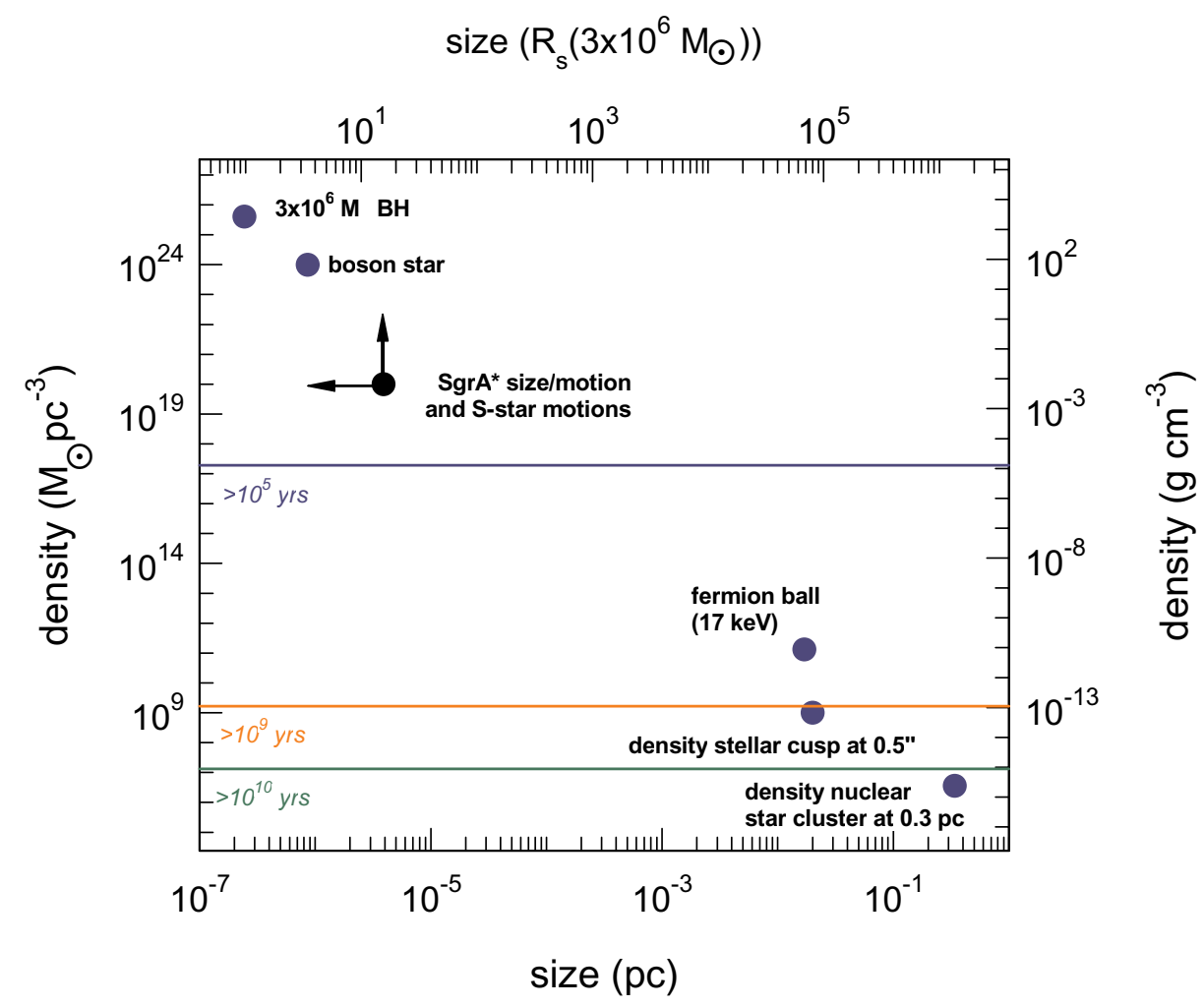

Figure 8. Constraints on the size (horizontal axis) and density (vertical axis) of Sgr A*. 1 pc corresponds to 3.26 light years. The circle with arrows denotes the observational constraints based on stellar orbits, radio size and the upper limit to Sgr A*'s proper motion. Filled circles denote various possible configurations of the mass. A black hole of 3 million $\mathrm{M}_{\odot}$ is located in the upper left corner, for which 'density' refers to the mass smeared out over the volume spanned by the event horizon. Top, middle and bottom horizontal lines mark the location of hypothetical dark astrophysical clusters (of neutron stars, white dwarfs, stellar black holes) of life-times $10^{5}$, $10^{9}$ and $10^{10}$ years, respectively.

spectroscopy with both the Keck and VLT shows that almost all of the cusp stars brighter than $K \simeq 16 \mathrm{mag}$ appear to be normal, main sequence B-type stars (Fig. 6, top central spectrum of S2; Ghez et al. 2003; Eisenhauer et al. 2005b). If these stars formed in situ also, the required cloud densities approach conditions in outer stellar atmospheres. Several scenarios have been proposed to account for this 'paradox of youth'. The most prominent are in situ formation in a dense gas accretion disk that can overcome the tidal limits, rapid in-spiral of a compact, massive star cluster that formed outside the central region, and re-juvenation of older stars by collisions or stripping (see Alexander 2005 for a detailed discussion and references). Based on the fact that most of the massive stars live in one of two fairly thin, rotating and coeval $(\sim 6 \mathrm{Myr})$ disks with a total mass of not much exceeding $10^{4} \mathrm{M}_{\odot}$, Paumard et al. (2006) conclude that the in situ star formation scenario is the likely answer for stars outside the central arcsecond, at least for the most prominent, so-called 'clockwise' disk. For B-type stars in the cusp immediately around Sgr A* the riddle remains unsolved, although most experts suspect some sort of efficient 


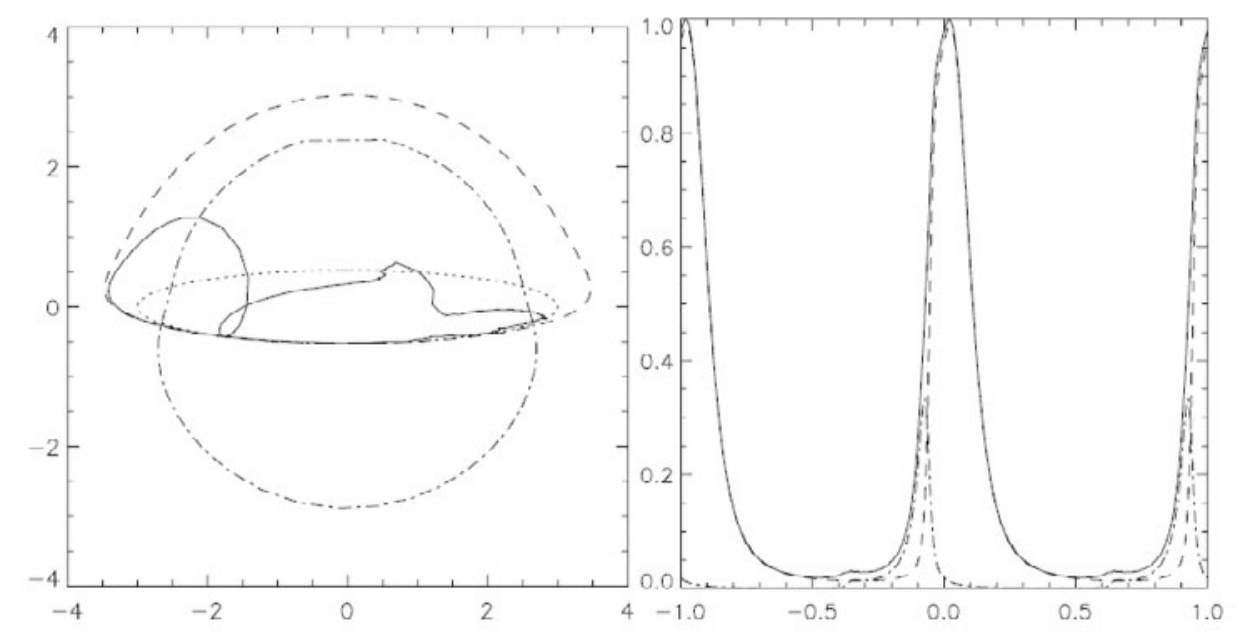

Figure 9. Photo-center wobbling (left) and light curve (right) of a hot spot on the innermost stable orbit around a Schwarzschild black hole, at an inclination of $80^{\circ}$ as derived from ray tracing in a Schwarzschild metric. Dotted curve: true path of the hot spot; dashed curves: apparent path and light curve of the primary image; dash-dotted curves: same for secondary image; solid curves: path of centroid and integrated light curve. Axes of left panel are in Schwarzschild radii of a 3 million $\mathrm{M}_{\odot}$ black hole, roughly equal to the astrometric accuracy of $10 \mu$-arcseconds. Abscissa axis of right panel is in cycles. The loop in the centroid's track is due to the secondary image, which is strongly sensitive to the space time. The overall motion can be detected at good significance at the anticipated accuracy of GRAVITY. Details can be obtained by analyzing several flares simultaneously (Gillessen et al. 2006b; Paumard et al. 2005).

transport, such as resonant relaxation and three-body collisions (Hopman \& Alexander 2006), with stripping of late-type stars as a possible dark horse (Davies \& King 2005).

\section{Compelling evidence for a central massive black hole}

With diffraction limited imagery starting in 1991 on the $3.5 \mathrm{~m}$ ESO New Technology Telescope and continuing since 2002 on the VLT, a group at MPE was able to determine proper motions of stars as close as $\sim 0.1^{\prime \prime}$ from Sgr A* (Eckart \& Genzel 1996, 1997). In 1995 a group at the University of California, Los Angeles started a similar program with the $10 \mathrm{~m}$ diameter Keck telescope (Ghez et al. 1998). Both groups independently found that the stellar velocities follow a 'Kepler' law $\left(v \propto R^{-1 / 2}\right)$ as a function of distance from Sgr A* and reach $\geqslant 10^{3} \mathrm{~km} \mathrm{~s}^{-1}$ within the central light month. This implies that the 3-4 million $\mathrm{M}_{\odot}$ found earlier by Prof. Townes and his group must be concentrated within this volume, making any configuration other than a massive black hole fairly unlikely.

Only a few years later both groups achieved the next and crucial step. They were able to determine individual stellar orbits for several of the stars very close to the compact radio source (Fig. 7; Schödel et al. 2002, 2003; Ghez et al. 2003, 2005a; Eisenhauer et al. 2005a). In addition to the astrometric imaging, they obtained near-diffraction limited 
Doppler spectroscopy of the same stars (Ghez et al. 2003; Eisenhauer et al. 2003a, 2005a), yielding precision measurements of the three dimensional structure of several orbits, as well as the distance to the Galactic Center (see below). At the time of writing, orbits have been determined for about a dozen stars in the central light month (Fig. 7). The central mass and most stellar orbital parameters derived by the two teams agree mostly very well. These orbits show that the gravitational potential indeed is that of a point mass centered on Sgr A* within the relative astrometric uncertainties of $\sim 10$ mas. The stars orbit the position of this dark mass like planets around the Sun. However, most orbits are fairly elliptical and their orientations appear to be random. Most of the mass must be concentrated well within the peri-approaches of the innermost stars, $\sim 10-20$ light hours, or 70 times the Earth orbit radius and about 1000 times the event horizon of a 3.6 million $\mathrm{M}_{\odot}$ black hole. There is presently no indication for an extended mass greater than about $5 \%$ of the point mass.

Simulations indicate that current measurement accuracies already are sufficient to detect the first and second order effects of Special and General Relativity (to $(v / c)^{2}$ ) in a few years time (Zucker et al. 2006). Observations with future $30 \mathrm{~m}+$ diameter telescopes should be able to measure the mass and distance to the Galactic Center to $\sim 0.1 \%$ precision, detect radial precession in stellar orbits due to General Relativity and constrain the extended mass to $<10^{-3}$ of the massive black hole (Weinberg et al. 2005). At that level a positive detection of a halo of stellar remnants (stellar black holes and neutron stars) and perhaps dark matter would appear to be likely. Future interferometric techniques will push capabilities yet further (see below).

As mentioned above the simultaneous measurement of positions/proper motions and radial velocities for a number of stars yields a direct ('primary') determination of the SunGalactic Center distance, $\mathrm{R}_{\circ}$. Eisenhauer et al. (2003a, 2005b), Eisenhouwer (in preparation), and Ghez et al. (in preparation) find $R_{\circ} \simeq 7.6 \mathrm{kpc}$, in good agreement with the values determined from other techniques (Reid 1993). While the statistical uncertainty of the inferred distance is only a few percent, superior to other methods, substantial systematic uncertainties presently dominate the error budget: a priori assumptions about the motion of Sgr A* itself, the weight given to the data sets near peri-approach (where flares from Sgr $\mathrm{A}^{*}$ have the greatest impact on the stellar positions) and the selection of stars included in the fitting procedure.

Long term VLBA-observations have set $2 \sigma$ upper limits of about $20 \mathrm{~km} \mathrm{~s}^{-1}$ and $2 \mathrm{~km} \mathrm{~s}^{-1}$ (or 50 micro-arcseconds per year!) to the motion of Sgr A* itself, along and perpendicular to the plane of the Milky Way, respectively (Fig. 3; Reid \& Brunthaler 2004; see also Backer \& Sramek 1999). This precision measurement demonstrates very clearly that the radio source itself must indeed be massive, with simulations indicating a lower limit to the mass of Sgr $\mathrm{A}^{*}$ of $\sim 10^{5} \mathrm{M}_{\odot}$. The intrinsic size of the radio source at millimeter wavelengths is less than 5 to 20 times the event horizon diameter (Fig. 3; Bower et al. 2004; Shen et al. 2005). Combining radio size and proper motion limit of Sgr A* with the dynamical measurements of the nearby orbiting stars leads to the conclusion that Sgr A* can only be a massive black hole, beyond any reasonable doubt (Fig. 8). An astrophysical dark cluster fulfilling the observational constraints would have a life-time less than a few $10^{4}$ years and thus can be safely rejected, as can be a possible 'fermion' ball of hypothetical heavy neutrinos. All configurations but a massive black hole and a hypothetical 'boson' star (which, however, is not stable when accreting baryons as in the Galactic Center) can be excluded by the available measurements (Schödel et al. 2003; Ghez et al. 2005a). Under the assumption of the validity of General Relativity, the Galactic Center is now the best quantitative evidence astrophysics has that (massive) black holes do indeed exist. 


\section{Zooming in on the event horizon}

Recent millimeter, infrared and X-ray observations have detected irregular, and sometimes intense outbursts of emission from Sgr A* lasting anywhere between 30 minutes and a number of hours and occurring at least once per day (Baganoff et al. 2001; Genzel et al. 2003a; Marrone et al. 2006). These flares originate from within a few milli-arcseconds of the radio position of Sgr A*. They probably occur when relativistic electrons in the innermost accretion zone of the black hole are significantly accelerated (from $\gamma \simeq 10$ to $\geqslant 10^{3}$ ) so that they are able to produce infrared synchrotron emission and X-ray synchrotron or inverse Compton radiation (Markoff et al. 2001; Yuan et al. 2003; Liu et al. 2005). This interpretation is also supported by the detection of significant polarization of the infrared flares (Eckart et al. 2006b), by the simultaneous occurrence of X-and IR-flaring activity (Eckart et al. 2006a; Yusef-Zadeh et al. 2006) and by variability in the infrared spectral properties (Ghez et al. 2005a; Gillessen et al. 2006a; Krabbe et al. 2006). There are indications for quasi-periodicities in the light curves of some of these flares, perhaps due to orbital motion of hot gas spots near the last circular orbit around the event horizon (Genzel et al. 2003a; Aschenbach et al. 2004; Belanger et al. 2006).

These and number other recent pan-chromatic observations of Sgr $\mathrm{A}^{*}$, in conjunction with increasingly detailed theoretical simulations, are also beginning to elucidate why Sgr $\mathrm{A}^{*}$ is so underluminous, $\sim 10^{-5}$ to $10^{-8}$ times the Eddington luminosity of a 3.6 million $\mathrm{M}_{\odot}$ black hole. It appears that the lack of radiation is due to a combination of several factors (Melia \& Falcke 2001). Only a small fraction $(\sim 0.1 \%$, Marrone et al. 2006 ) of the gas apparently on its way to the black hole at the Bondi radius of $\sim 1^{\prime \prime}$ $\left(\sim 10^{-5} \mathrm{M}_{\odot} \mathrm{yr}^{-1}\right.$, Quataert 2004$)$ actually penetrates to the event horizon. The rest is probably ejected back out because of inefficient angular momentum transport and a strong outflow from the inner accretion zone (Blandford \& Begelman 1999; De Villier et al. 2005). In addition, the accretion flow is probably radiatively inefficient, due to poor thermal coupling between the electrons and the ions (Narayan et al. 1996; Yuan et al. 2003). Finally, the accretion rate and luminosity of Sgr A* may be strongly time-variable on time scales of a few hundred years (Revnivtsev et al. 2004).

The infrared flares as well as the steady microwave emission from $\mathrm{Sgr} \mathrm{A}^{*}$ may be important probes of the dynamics and space time around the black hole (Broderick \& Loeb 2006). Future long-baseline interferometry at short millimeter or submillimeter wavelengths may be able to map out the strong light bending ('shadow') around the photon orbit of the black hole (Falcke et al. 2000). Eisenhauer et al. (2005b) are developing 'GRAVITY' for the VLT-Interferometer, which is intended to yield dual-beam, 10 microarcsecond precision infrared astrometric imaging of faint sources. GRAVITY may be able to map out the motion on the sky of hot spots during flares with a high enough resolution and precision to determine the size of the emission region and possibly detect the imprint of multiple gravitational images (Fig. 9; Paumard et al. 2005; Gillessen et al. 2006b). In addition to studies of the flares, GRAVITY will also be able to image the orbits of stars very close to the black hole, which should then show radial and Lense-Thirring orbital precessions due to General Relativity. Both the microwave 'shadows' as well as the infrared hot spots are sensitive to the space time and metric in the strong gravity regime (Broderick \& Loeb 2006). As such, these ambitious future experiments can potentially test the validity of the black hole model near the event horizon and perhaps even the validity of General Relativity in the strong field limit.

\section{References}

Aharonian, F., Akhperjanian, A.G., Aye, K.-M., et al. 2004, A\&̈A (Letters), 425, L13 
Alexander, T. 2005, Phys. Rep., 419, 65

Allen, D.A., \& Sanders, R. 1986, Nature, 319, 191

Allen, D.A., Hyland, A.R., \& Hillier, D.J. 1990, MNRAS, 244, 706

Aschenbach, B., Grosso, N., Porquet, D., \& Predehl, P. 2004, A\&3A, 417, 71

Backer, D.C., \& Sramek, R.A. 1999, ApJ, 524, 805

Baganoff, F., Bautz, M.W., Brandt, W.N., et al. 2001, Nature, 413, 45

Balick, B., \& Brown, R. 1974, ApJ, 194, 265

Beckers, J.M. 1993, Ann. Rev. A\&SA, 31, 13

Becklin, E.E., \& Neugebauer, G. 1975, ApJ, 200, L71

Becklin, E.E., Matthews, K., Neugebauer, G., \& Willner, S.P. 1978, ApJ, 219, 121

Belanger, G., Terrier, R., de Jager, O.C., Goldwurm, A., \& Melia, F. 2006, J.Phys.CS, 54, 420

Blandford, R., \& Begelman, M.C. 1999, MNRAS (Letters), 303, L1

Bonnet, H., Abuter, R., Baker, A., et al. 2004, ESO Messenger, 117, 17

Bower, G.C., Falcke, H., Herrnstein, R.M., et al. 2004, Science, 304, 704

Broderick, A., \& Loeb, A. 2006, J.Phys.CS, 54, 448

Davies, M.B., \& King, A. 2005, ApJ (Letters), 624, L25

DePoy, D.L., \& Sharp, N.A. 1991, AJ, 101, 1324

De Villier, J.P. Hawley, J.F., Krolik, J.H., \& Hirose, S. 2005, ApJ, 620, 878

Eckart, A., Genzel, R., Hofmann, R., Sams, B.J., \& Tacconi-Garman, L.E. 1993, ApJ (Letters), 407, L77

Eckart, A., Genzel, R., Hofmann, R., Sams, B.J., \& Tacconi-Garman, L.E. 1995, ApJ (Letters), $445, \mathrm{~L} 23$

Eckart, A., \& Genzel, R. 1996, Nature, 383, 415

Eckart, A., \& Genzel, R. 1997, MNRAS, 284, 576

Eckart, A., Baganoff, F.K., Schödel, R., et al. 2006a, A\&A, 450, 535

Eckart, A., Schödel, R., Meyer, L., et al. 2006b, A\&̈A, 455, 1

Einstein, A. 1916, Ann. Phys., 49, 50

Eisenhauer, F., Schödel, R., Genzel, R., et al. 2003a, ApJ (Letters), 597, L121

Eisenhauer, F., Abuter, R., Bickert, K., et al. 2003b, Proc. SPIE, 4841, 1548

Eisenhauer, F., Genzel, R., Alexander, T., et al. 2005a, ApJ, 628, 246

Eisenhauer, F., Perrin, G., Rabien, S., Eckart, A., Lena, P., Genzel, R., Abuter, R., \& Paumard, T. 2005b, Astron. Nachr., 326, 561

Falcke, H., Melia, F., \& Algol, E. 2000, ApJ (Letters), 528, L13

Ferrarese, L., \& Merritt, D. 2000, ApJ (Letters), 539, L9

Forrest, W.J., Shure, M.A., Pipher, J.L., \& Woodward, C.A. 1987, in: D.C. Backer (ed.), The Galactic Center, Proc. Symp. honoring C.H. Townes, Berkeley, CA, USA, 1986, AIP-CP, 155,153

Genzel, R., Hollenbach, D., \& Townes, C.H. 1994, Rep. Prog. Phys., 57, 417

Genzel, R., Schödel, R., Ott, T., et al. 2003a, Nature, 425, 934

Genzel, R., Schödel, R., Ott, T., et al. 2003b, ApJ, 594, 812

Ghez, A.M., Klein, B.L., Morris, M., \& Becklin, E.E. 1998, ApJ, 509, 678

Ghez, A.M., Duchêne, G., Matthews, K., et al. 2003, ApJ (Letters), 586, L127

Ghez, A.M., Salim, S., Hornstein, S.D., et al. 2005a, ApJ, 620, 744

Ghez, A.M., Hornstein, S.D., Lu, J.R., et al. 2005b, ApJ, 635, 1087

Giacconi, R., Gursky, H., Paolini, F., \& Rossi, B.B. 1962, Phys. Rev. Lett., 9, 439

Giacconi, R. 2003, Rev. Mod. Phys., 75, 995

Gillessen, S., Eisenhauer, F., Quataert, E., et al. 2006a, ApJ (Letters), 640, L163

Gillessen, S., Perrin, G., Brandner, W., et al. 2006b, in: J.D. Monnier, M. Schöller \& W.C. Danchi (eds.), Advances in Stellar Interferometry, SPIE, 6268, 33

Hopman, C., \& Alexander, T. 2006, ApJ, 645, 1152

Kerr, R. 1963, Phys. Rev. Lett., 11, 237

Kormendy, J. 2004, in: L.C. Ho (ed.), Coevolution of Black Holes and Galaxies, Proc. Carnegie Observatories Astrophysics Series (Cambridge: CUP), p. 1

Krabbe, A., Iserlohe, C., Larkin, J.E., et al. 2006, ApJ (Letters), 642, L145 
Lenzen, R., Hofmann, R., Bizenberger, P., \& Tusche, A. 1998, in: A.M. Fowler (ed.), Infrared Astronomical Instrumentation, Proc. SPIE, 3354, 606

Liu, S., Melia, F., \& Petrosian, V. 2005, ApJ, 636, 798

Lynden-Bell, D. 1969, Nature, 223, 690

Markoff, S., Falcke, H., Yuan, F., \& Biermann, P.L. 2001, A $\& A$ (Letters), 379, L13

Marrone, D., Moran, J.M., Zhao, J.-H., \& Rao, R. 2006, ApJ, 640, 308

Matthews, K., et al. 2007, in preparation

Melia, F., \& Falcke, H. 2001, Ann. Rev. A\&A, 39, 309

McClintock, J.E., \& Remillard, R.A. 2006, in: W. Lewin \& M. van der Klis (eds.), Compact Stellar X-ray Sources, Cambridge Astrophysics Series No. 39. (Cambridge: CUP), p. 157.

Miyoshi, M., Moran, J., Herrnstein, J., et al. 1995, Nature, 373, 127

Morris, M. 1993, ApJ, 408, 496

Narayan, R., Yi, I., \& Mahadevan, R. 1996, Nature, 374, 623

Paumard, T., Perrin, G., Eckart, A., Genzel, R., Lena, P., Schödel, R., Eisenhauer, F., Müller, T., \& Gillessen, S. 2005, Astron. Nachr., 326, 568

Paumard, T., Genzel, R., Martins, F., et al. 2006, ApJ, 643, 1011

Quataert, E. 2004, ApJ, 613, 322

Rees, M. 1984, Ann. Rev A\&A, 22, 471

Reid, M.J. 1993, Ann. Rev. A\& A, 31, 345

Reid, M.J., \& Brunthaler, A. 2004, ApJ, 616, 872

Revnivtsev, M.G., Churazov, E.M., Sazonov, S.Yu., et al. 2004, A\&A (Letters), 425, L49

Rivera, E.J., Lissauer, J.J., Butler, R.P., et al. 2005, ApJ, 634, 625

Roberts, D.A., \& Goss, W.M. 1993, ApJS, 86, 133

Rousset, G., Lacombe, F., Puget, P., et al. 1998, in: D. Bonaccini \& R.K. Tyson (eds.), Adaptive Optical System Technologies, Proc. SPIE, 3255, 508

Schödel, R., Ott, T., Genzel, R., et al. 2002, Nature, 419, 694

Schödel, R., Ott, T., Genzel, R., et al. 2003, ApJ, 596, 1015

Schödel, R., Eckart, A., Muzic, K., et al. 2007, A\& A (Letters), 462, L1

Schmidt, M. 1963, Nature, 197, 1040

Schwarzschild, K. 1916, Sitzungsber. Preuss. Akad. Wiss., 424

Shen, Z.Q., Lo, K.Y., Liang, M.C., Ho, P.T.P., \& Zhao, J.H. 2005, Nature, 438, 62

Townes, C.H., Lacy, J.H., Geballe, T.R., \& Hollenbach, D.J. 1982, Nature, 301, 661

Weinberg, N.N., Milosavljevic, M., \& Ghez, A.M. 2005, ApJ, 622, 878

Wheeler, J.A. 1968, American Scientific, 56, 1

Wizinovich, P., et al. 2007, in preparation

Wollman, E.R., Geballe, T.R., Lacy, J.H., Townes, C.H., \& Rank, D.M. 1977, ApJ (Letters), 218, L103

Yuan, F., Quataert, E., \& Narayan, R. 2003, ApJ, 598, 301

Yusef-Zadeh, F., Bushouse, H., Dowell, C.D., et al. 2006, ApJ, 644, 198

Zucker, S., Alexander, T., Gillessen, S., Eisenhauer, F., \& Genzel, R. 2006, ApJ (Letters), 639, L21 